\section{Real-world management of juvenile autoimmune liver disease}

\author{
Y S de Boer ${ }^{1} \oplus$, Liberal $^{2}$, D Vergani ${ }^{2}$ and G Mieli-Vergani ${ }^{2,3}$; \\ on behalf of the International Autoimmune Hepatitis Group (IAIHG)
}

United European Gastroenterology Journal 2018, Vol. 6(7) 1032-1038 (C) Author(s) 2018 Reprints and permissions: sagepub.co.uk/journalsPermissions.nav DOI: $10.1177 / 2050640618768922$ journals.sagepub.com/home/ueg @SAGE

\begin{abstract}
Background and aims: Juvenile autoimmune liver disease (JAILD) includes paediatric forms of autoimmune hepatitis (AIH) and autoimmune sclerosing cholangitis (ASC). Since evidence is scarce, there are currently no evidence-based management guidelines for juvenile AlH. This survey was carried out amongst the paediatric members of the International AlH Group (IAIHG) to describe their practices in the management of JAILD.

Methods: An online survey questionnaire was distributed to members of the IAIHG with active practice (https://www. surveymonkey.de/r/Juvenile_AILD). The questionnaire consisted of four clinical scenarios on different presentations of AlH. Results: Fifty-eight surveys were sent to the IAIHG members, out of which 43 (74\%, 22 countries, four continents) were returned. None reported budesonide as a first-line induction agent for the acute presentation of AlH. Sixteen ( $37 \%$ ) routinely perform liver biopsy at three years of biochemical remission. Thirty-five respondents (81\%) perform magnetic resonance cholangiography (MRC) at presentation. Ciclosporin is the most widely used second-line agent (number of patients treated $=\sim 360,21$ centres). Mycophenolate mofetil ( $n=\sim 225,31$ centres), tacrolimus ( $n=\sim 130,21$ centres) and sirolimus ( $n=\sim 5,3$ centres) are less often reported. Rescue therapy with infliximab and rituximab has been tried in eight centres $(n=\sim 19)$ and nine centres $(n=\sim 16)$, respectively.

Conclusions: Prednisolone remains the preferred first-line induction agent in JAILD. MRC at presentation is performed by the large majority of participants. Participants reported a wide variation in performing liver biopsy for therapy evaluation during follow-up. Within the paediatric members of the IAIHG there is considerable experience with second-line therapeutic agents.
\end{abstract}

\title{
Keywords
}

Autoimmune hepatitis, autoimmune sclerosing cholangitis, immunosuppression, liver transplantation, International Autoimmune Hepatitis Group

Received: 16 February 2018; accepted: 12 March 2018

\section{Key summary}

- Prednisolone remains the preferred first-line induction agent in juvenile autoimmune liver disease.

- Magnetic resonance cholangiography at presentation of juvenile autoimmune liver disease is performed by the large majority of expert paediatric hepatologists.

- There is considerable experience with second-line therapeutic agents among the paediatric hepatologist members of the international autoimmune hepatitis group.

\footnotetext{
${ }^{1}$ Department of Gastroenterology and Hepatology, VU University Medical Center, The Netherlands

${ }^{2}$ Institute of Liver Studies, King's College London, UK

${ }^{3}$ Paediatric Liver, GI and Nutrition Centre, King's College Hospital, UK
}

Y.S.d.B. and R.L. contributed equally to this work.

Corresponding author:

Y.S. de Boer, Department of Gastroenterology and Hepatology, VU University Medical Center Amsterdam, Boelelaan 1118, 1081 HV Amsterdam, The Netherlands.

Email: y.deboer@vumc.nl 


\section{Introduction}

Juvenile autoimmune liver disease (JAILD) includes paediatric forms of autoimmune hepatitis (AIH) and autoimmune sclerosing cholangitis (ASC). ${ }^{1}$ While the target of the autoimmune attack in AIH are the hepatocytes, in ASC, the biliary epithelial cells lining the bile ducts are also targeted. ${ }^{2}$ AIH is a severe lifethreatening chronic progressive inflammatory liver disorder characterised by high levels of transaminases and immunoglobulin $\mathrm{G}$ ( $\mathrm{IgG}$ ), presence of autoantibodies, and histologically by interface hepatitis. ${ }^{3}$ In children and adolescents, AIH often presents acutely and has a more aggressive course than in middle-age and elderly patients. $^{4,5}$ On the basis of the type of antibodies detected at the time of diagnosis, two forms of AIH are recognised: autoimmune hepatitis type 1 (AIH-1) defined by the presence of antinuclear antibody (ANA) and/or anti-smooth muscle antibody (SMA), and autoimmune hepatitis type 2 (AIH-2), which is characterised by positivity for anti-liver kidney microsomal type 1 (anti-LKM-1) and/or anti-liver cytosol type 1 (anti-LC-1) antibodies. ${ }^{6}$ Although AIH-1 affects children and adults, AIH-2 is predominantly a paediatric condition accounting for one-third of juvenile patients. ${ }^{4}$ Sclerosing cholangitis is a chronic cholestatic disorder characterised by progressive inflammation and fibrosis of intrahepatic and/or extrahepatic bile ducts that leads to bile duct obliteration with formation of multifocal bile duct strictures. ${ }^{7}$ The term primary sclerosing cholangitis (PSC) used in adult hepatology is not accurate to define paediatric sclerosing cholangitis because in children, sclerosing cholangitis is often associated with florid autoimmune features such as elevated titres of autoantibodies, elevated $\mathrm{IgG}$, and interface hepatitis on histology. ${ }^{8,9}$ This condition, often associated with inflammatory bowel disease, is referred to as autoimmune sclerosing cholangitis (ASC) and is as prevalent as AIH-1 in childhood, but in contrast to AIH it affects equally boys and girls. ${ }^{8}$ There is no single diagnostic test for AIH or ASC, and diagnosis is based on several indicative clinical, biochemical, serological and histological findings; in addition, diagnosis of ASC requires cholangiography. ${ }^{10}$ The International Autoimmune Hepatitis Group (IAIHG) has devised two AIH diagnostic scoring systems for adult patients, the revised original (1999) and the simplified (2008) criteria. ${ }^{11,12}$ However, neither scoring system is suitable for the juvenile form of the disease, in which diagnostically relevant autoantibodies often have titres lower than the cut-off value considered positive in adults. ${ }^{10} \mathrm{In}$ addition, neither system can distinguish between AIH and ASC, which can be differentiated only if a cholangiogram is performed at presentation. ${ }^{8}$ Although relatively rare, JAILDs are devastating diseases which progress rapidly unless immunosuppressive treatment is started promptly. ${ }^{1}$ The treatment of JAILD is still largely based on the results of randomised trials in adult AIH patients published four decades ago. Since evidence is scarce, there are currently no evidence-based management guidelines for paediatric AIH or ASC, despite the guidelines published by the American Association for the Study of the Liver (AASLD) and the European Association for the Study of the Liver (EASL). ${ }^{13,14}$ In addition, decisions regarding the use of second-line therapies are based on small series or even case reports, mostly reporting the experience of a limited number of centres with a special interest in JAILD. ${ }^{10}$ A recent European Society of Paediatric Gastroenterology, Hepatology and Nutrition (ESPGHAN) position statement addresses diagnostic and management issues specifically related to JAILD. ${ }^{15}$ The present study was designed to explore the current practices on the diagnosis, treatment and follow-up of patients with JAILD of a panel comprising international expert paediatric hepatologists in order to help design and inform future prospective studies.

\section{Methods}

\section{Study design}

We developed a survey questionnaire to assess the practices of an international panel of expert paediatric hepatologists on the clinical management of JAILD. The participants were enlisted if the following criteria were met: membership in the IAIHG, active practice of paediatric patients with AILD, and expertise in the field based on a relevant track record of publications. The survey was made available online, and an email link to the survey was sent to 58 experts in September 2016, followed by a total of four weekly reminders. Participants were asked to provide details on their clinical practice: number of years in practice, centre, country, approximate number of JAILD patients, whether working at a transplant centre. Participation was on a voluntary basis and was not compensated.

\section{Questionnaire}

The questionnaire consisted of four clinical scenarios on different presentations of JAILD in which 30 questions were asked (supplementary material, (https://www.surveymonkey.de/r/Juvenile_AILD). Briefly, cases consisted of a succinct history and results from diagnostic work-up, in short representing an acute presentation of a 6-year-old girl (Case 1), presentation and management of a 13-year-old boy with ASC (Case 2), management and follow-up of a 15-year-old boy with AIH not responding to standard therapy requiring second-line 
agents (Case 3 ), and a 16 year-old boy with minocycline-induced AIH (Case 4). Answers to the provided questions were offered as integer multiple choice, allowing for a free-text alternative (other).

\section{Data presentation and analysis}

Data were collected non-anonymously and analysed using the graphical and analytical features of www.surveymonkey.com or IBM SPSS Statistics 22. Answers are described as counts and percentages for categorical variables.

\section{Ethical considerations}

This study was conducted according to the Declaration of Helsinki. All authors reviewed and approved the final manuscript.

\section{Results}

\section{Participants}

A total of 58 surveys were sent to the IAIHG members fulfilling the criteria mentioned above, out of which $43(74 \%)$ were returned. All 43 respondents answered every question. Twenty-two countries from four different continents were represented. The number of JAILD patients treated by the participating physicians ranged from $<20$ in $11(26 \%)$ to $>200$ in six $(14 \%)$ centres. Twenty-five respondents $(68 \%)$ had $>20$ years of experience and $24(65 \%)$ were active in a transplant centre. There were no differences in terms of number of JAILD patients or years of experience between respondents working at transplant vs. non-transplant centres. Table 1 summarises the characteristics of respondents.

\section{First-line therapy of AlH: Induction, maintenance and withdrawal}

Forty respondents $(93 \%)$ initiate treatment with predniso(lo)ne, one $(2 \%)$ with methylprednisolone and two $(5 \%)$ with tacrolimus in isolation in the acute presentation of AIH. None reported budesonide as a first-line induction agent. Five (12\%) participants use predniso(lo)ne in isolation without adding azathioprine (AZA) at initiation or follow-up. Ten (23\%) would simultaneously add AZA at $1-1.5 \mathrm{mg} / \mathrm{kg} / \mathrm{day}$ to the steroid therapy, whereas the majority $(n=26)$ would subsequently introduce AZA maintenance therapy while tapering steroids. Overall, 21 participants (49\%) reported performing thiopurine S-methyltransferase activity before the initiation of thiopurine therapy. The preferred daily induction dose of predniso(lo)ne ranged from $1 \mathrm{mg} / \mathrm{kg} /$ day reported by 20 participants $(47 \%)$ to $2 \mathrm{mg} / \mathrm{kg} /$ day by nine $(21 \%)$. Ten participants $(23 \%)$ reported a standardised induction dose of predniso(lo)ne of $40 \mathrm{mg} /$ day. All participants would taper predniso(lo)ne dose over the next two to three months; the majority would aim for a minimal possible dose $(n=20)$ or a daily dose of $5 \mathrm{mg} /$ day $(n=11)$. Twenty-two $(51 \%)$ participants reported the routine use of 6-thioguanine nucleotide level measurements to monitor adherence to thiopurine therapy. Thirty $(70 \%)$ performed routine measurements of autoantibody titres during follow-up. Twenty-seven participants (63\%) routinely perform a dual-energy X-ray absorptiometry scan to check for the development of osteoporosis during follow-up. In Case 1, 20 participants (48\%) would have performed liver biopsy when the patient was in stable biochemical remission to evaluate potential drug withdrawal. If histological inflammation and severe fibrosis or cirrhosis were absent in the treatment evaluation biopsy, $14(33 \%)$ participants would attempt prednisolone withdrawal, one $(2 \%)$ would attempt AZA withdrawal, whereas five $(12 \%)$ would attempt withdrawal of both drugs.

\section{Diagnosis and management of ASC}

At presentation of JAILD, 35 respondents (81\%) perform magnetic resonance cholangiography (MRC) to rule out sclerosing cholangitis, whereas one participant reported endoscopic retrograde cholangiography as the preferred modality. If ASC is documented, participants perform a colonoscopy to rule out inflammatory bowel disease (IBD) if the patient is symptomatic $(n=6)$ and/ or has raised calprotectin levels $(n=14)$ or even in the absence of symptoms $(n=20)$.

\section{Second-line therapy}

The respondents were asked to provide the approximate number of patients that were treated with six medications that are considered as second-line therapy by Society guidelines. Overall, the large majority of the participating paediatric hepatologists $(n=37,86 \%)$ reported to have some experience with second-line medication in the management of AIH. Ciclosporin is the most widely used second-line agent (number of patients treated $=\sim 360,21$ centres). Mycophenolate mofetil (MMF) ( $n=\sim 225,31$ centres), tacrolimus $(n=\sim 130$, 21 centres) and sirolimus $(n=\sim 5$, three centres) are less often reported (Figure 1). Rescue therapy with infliximab and rituximab has been tried in eight centres $(n=\sim 19)$ and nine centres $(n=\sim 16)$, respectively (Figure 1). Most of the experience with second-line therapy using ciclosporin and tacrolimus resides in the larger tertiary referral centres with a transplant programme, 
Table 1. Characteristics of respondents.

\begin{tabular}{|c|c|c|c|c|}
\hline Country & $\begin{array}{l}\text { Years in } \\
\text { practice }\end{array}$ & $\begin{array}{l}\text { Number of paediatric } \\
\text { AlH patients in centre }\end{array}$ & $\begin{array}{l}\text { Number of adult AlH } \\
\text { patients in centre }\end{array}$ & $\begin{array}{l}\text { Transplant } \\
\text { centre }\end{array}$ \\
\hline Argentina & $>20$ & $>200$ & & Yes \\
\hline Argentina & $>20$ & $>200$ & & Yes \\
\hline Argentina & $>20$ & $>200$ & $<20$ & Yes \\
\hline Argentina & $>20$ & $20-50$ & & No \\
\hline Austria & $<5$ & $20-50$ & & No \\
\hline Belgium & $>20$ & $20-50$ & & Yes \\
\hline Brazil & $>20$ & $100-200$ & $>200$ & Yes \\
\hline Canada & $>20$ & $50-100$ & & Yes \\
\hline Canada & $10-20$ & $<20$ & $<20$ & No \\
\hline Canada & $>20$ & $20-50$ & & No \\
\hline Canada & $10-20$ & $50-100$ & $<20$ & Yes \\
\hline Chile & $5-10$ & $<20$ & & Yes \\
\hline Denmark & $10-20$ & $20-50$ & $100-200$ & Yes \\
\hline France & $10-20$ & $<20$ & & No \\
\hline France & $>20$ & $50-100$ & $<20$ & Yes \\
\hline France & $>20$ & $20-50$ & & Yes \\
\hline Germany & $>20$ & $50-100$ & $>200$ & Yes \\
\hline Germany & $>20$ & $20-50$ & & Yes \\
\hline Germany & $10-20$ & $50-100$ & $>200$ & Yes \\
\hline Israel & $>20$ & $<20$ & $<20$ & No \\
\hline Italy & $>20$ & $50-100$ & $<20$ & No \\
\hline Italy & $10-20$ & $20-50$ & & Yes \\
\hline Italy & $>20$ & $<20$ & & No \\
\hline Italy & $5-10$ & $20-50$ & $<20$ & No \\
\hline Japan & $>20$ & $20-50$ & $<20$ & No \\
\hline Poland & $>20$ & $>200$ & & Yes \\
\hline Portugal & $>20$ & $<20$ & & No \\
\hline Portugal & $>20$ & $20-50$ & & Yes \\
\hline Romania & $10-20$ & $<20$ & & No \\
\hline Spain & $>20$ & $50-100$ & & Yes \\
\hline Sweden & $>20$ & $50-100$ & $50-100$ & Yes \\
\hline Switzerland & $10-20$ & $<20$ & $<20$ & No \\
\hline Switzerland & $10-20$ & $<20$ & & Yes \\
\hline Taiwan & $10-20$ & $<20$ & $<20$ & Yes \\
\hline Turkey & $10-20$ & $20-50$ & $20-50$ & Yes \\
\hline Turkey & $10-20$ & $20-50$ & $50-100$ & Yes \\
\hline United Kingdom & $>20$ & $>200$ & $>200$ & Yes \\
\hline United Kingdom & $>20$ & $>200$ & $<20$ & Yes \\
\hline United States & $5-10$ & $100-200$ & & Yes \\
\hline United States & $>20$ & $<20$ & & Yes \\
\hline United States & $>20$ & $50-100$ & & Yes \\
\hline United States & $10-20$ & $20-50$ & $50-100$ & Yes \\
\hline United States & $>20$ & $20-50$ & & Yes \\
\hline
\end{tabular}




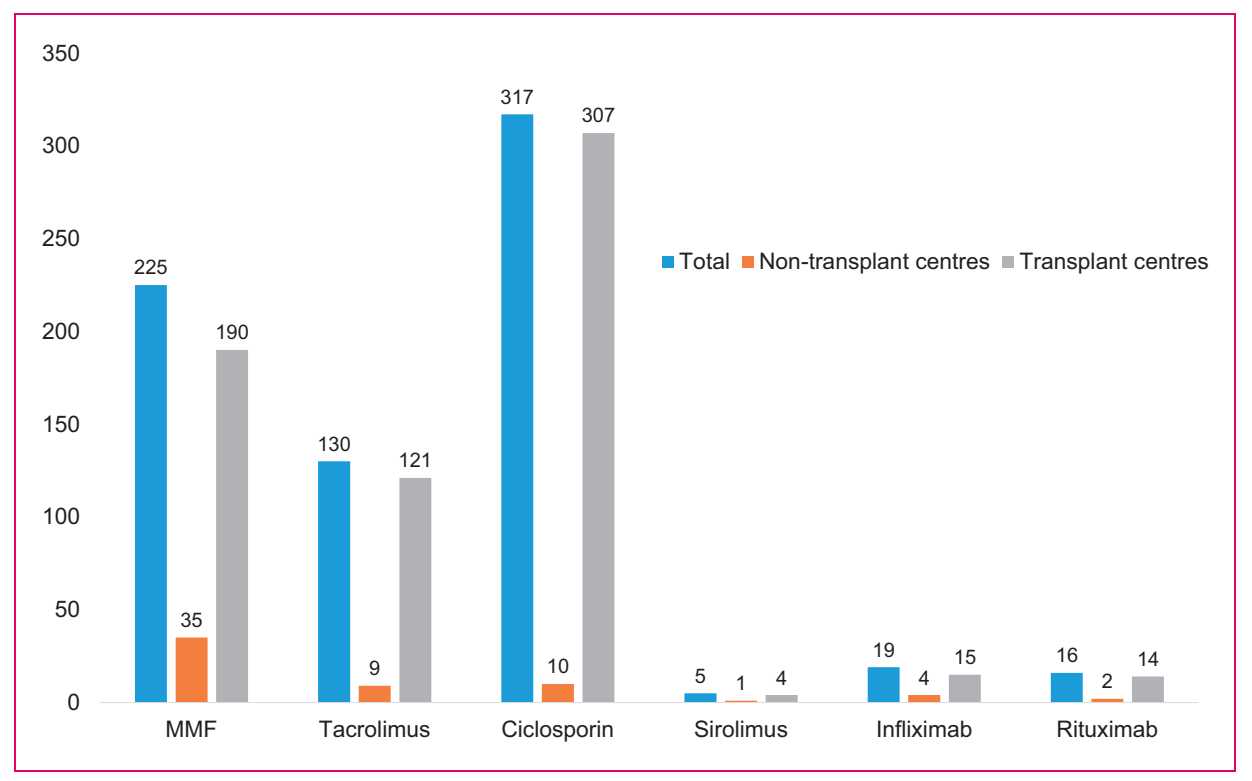

Figure 1. Reported number of paediatric patients treated with second-line therapies in the centres of participating paediatricians. MMF: mycophenolate mofetil.

but these agents are also used in small numbers of patients in non-transplant centres (Figure 1).

\section{Approach to suspected DILI with autoimmune features}

A majority of 25 participants $(58 \%)$ would treat a patient with severe autoimmunity like hepatitis following minocycline ingestion, prescribed for severe acne, with steroids in addition to discontinuation of the minocycline. Thirteen $(30 \%)$ would carefully monitor the patient and start immunosuppression only in case of progression of disease. Interestingly, one participant would treat this patient with tacrolimus monotherapy, aiming for a combined beneficial effect on the hepatitis and acne. At three months after initiation of prednisolone therapy and a rapid normalisation of laboratory parameters, 12 participants $(28 \%)$ would discontinue prednisolone, and five $(12 \%)$ would perform repeat biopsy to evaluate inflammatory activity before discontinuation of prednisolone. However, the majority $(n=22,52 \%)$ would continue prednisolone for another three months before discontinuation $(n=7,17 \%)$ or would perform a repeat biopsy after six months of treatment to evaluate its efficacy $(n=15,36 \%)$. At complete normalisation after six months and discontinuation of prednisolone, one participant would discharge the patient, whereas the large majority suggested a follow-up monthly $(n=6,14 \%)$, three-monthly $(n=24,57 \%)$, six-monthly $(n=7,17 \%)$ or yearly $(n=2,5 \%)$. In case of a flare with elevated transaminases and hyperbilirubinemia during followup, 16 participants $(38 \%)$ would restart therapy with high-dose $(n=6,14 \%)$ or low-dose prednisolone $(n=10,24 \%)$ therapy, whereas $24(57 \%)$ participants would first perform a liver biopsy and treat the patient depending on histological inflammatory activity. Twenty-four participants would subsequently add AZA $(n=23,55 \%)$ or tacrolimus $(n=1,2 \%)$ as a maintenance agent and $15(36 \%)$ would keep the patient on monotherapy with prednisolone.

\section{Discussion}

This survey shows that prednisolone remains the preferred agent for induction of remission in newly diagnosed paediatric patients with AIH, but there is a lack of consensus among expert paediatric hepatologists regarding both the initial management and follow-up of patients with AIH. In addition, and despite the lack of good quality evidence, there is considerable experience, albeit largely unreported, in relation to second- and third-line therapies for difficult-to-treat AIH patients. All but two of the experts surveyed routinely use prednisolone as initial treatment for AIH, but there is a wide variation in the dose and time that is taken to taper the dose. While a minority of respondents starts therapy with a combination of prednisolone and AZA, the majority starts prednisolone in isolation adding AZA only once steroids are being tapered. These different therapeutic approaches reflect the lack of evidencedbased protocols for the treatment of paediatric $\mathrm{AIH}$, a subject only briefly discussed in the AASLD ${ }^{13}$ and EASL $^{14}$ guidelines. For this reason, recently the ESPGHAN Hepatology Committee has issued a position paper based on published paediatric series in order to addresses diagnostic and management issues 
specifically related to the juvenile form of the disease. ${ }^{15}$ The position statement suggests starting treatment with prednisolone (or prednisone) $2 \mathrm{mg} / \mathrm{kg} /$ day (maximum $60 \mathrm{mg} /$ day), and decreasing it gradually over a period of four to eight weeks in parallel to the decline of transaminase levels to achieve a maintenance dose of 2.5 to $5 \mathrm{mg} /$ day. In regards to the addition of AZA, different protocols are used by different centres, but, in view of the potential hepatotoxicity of AZA in the presence of jaundice and/or cirrhosis, the position paper suggests introducing it gradually, if transaminase levels plateau or increase while decreasing prednisolone, aiming to a final dose of $1-2 \mathrm{mg} / \mathrm{kg} /$ day. Alternatively, AZA could be added per protocol after two weeks of predniso(lo)ne treatment, when the disease is less active. A similar delay in introducing AZA treatment has been proposed also for adult patients in the EASL guidelines. ${ }^{14}$ Whether this strategy offers an advantage over the simultaneous use of prednisolone and AZA at disease presentation is unknown, since studies addressing this question are currently not available. Interestingly, none of the respondents reported the use of budesonide as a first-line agent for induction of remission. In a recent multicentre European trial, which included 46 treatment-naïve children, a combination of budesonide with AZA was compared with a combination of prednisolone with AZA. ${ }^{16}$ At six months the remission rate was $16 \%$ in the budesonide arm vs $15 \%$ in the prednisolone arm, while at 12 months it was $50 \%$ and $42 \%$, respectively. ${ }^{17}$ Since these rates are much lower compared to those achieved with the standard treatment, the recent ESPGHAN position statement stresses that at present time an induction strategy with budesonide does not offer an advantage over the standard treatment. ${ }^{15}$ At presentation of a child with $\mathrm{AIH}$, the vast majority of participants reported performing a cholangiogram in order to rule out overlap with sclerosing cholangitis, a trend reflecting both EASL guidelines and the recent ESPGHAN position statement. ${ }^{14,15}$ Indeed, the only prospective study published to date showed that when cholangiographic studies are performed the prevalence of ASC is similar to that of $\mathrm{AIH}^{8}{ }^{8}$ On the other hand, only a small majority of respondents performs a colonoscopy to rule out IBD in the absence of symptoms, whereas the rest would perform this only in the presence of clinical symptoms or an elevated faecal calprotectin level, testing for which is advised in all JAILDs in the ESPHGAN position statement. ${ }^{15}$ For patients who are refractory to standard therapy, proposed alternative treatments are based on scarce published data. Nevertheless, this study shows that amongst paediatric experts there is ample experience with second-line agents, and indeed more than among the IAIHG adult hepatologist members. ${ }^{18} \mathrm{~A}$ recent meta-analysis, including 76 children refractory to standard therapy, reported a high response rate at six months with calcineurin inhibitors, although this response was burdened with a high incidence of adverse events. ${ }^{19}$ MMF was the second most effective drug with a low side effect profile, ${ }^{19}$ supporting the notion that MMF should be the primary choice for second-line therapy in AIH children refractory to standard treatment, as also advised by the ESPGHAN position statement. ${ }^{15}$ It should be noted that MMF has an established teratogenic potential and therefore it should be prescribed with caution in juvenile female patients who may become pregnant. ${ }^{20,21}$ Only in case of non-response or intolerance to MMF should the use of calcineurin inhibitors be considered. ${ }^{15}$ Although minocycline is commonly prescribed in adolescents for the treatment of severe acne vulgaris, this is not without risk of severe side effects, also compared to other tetracyclines. ${ }^{22}$ The drug has been associated with development of drug reaction and systemic symptoms syndrome, several autoimmune diseases (including systemic lupus erythematosus) as well as severe hepatotoxicity with cirrhosis development, both in children and adults. ${ }^{22-24}$ Whether the liver injury reported is the result of minocycline-induced AIH or whether this denotes an episode of self-limiting druginduced liver injury with concomitant autoimmune features is not self-evident at the presentation of such a complication, but this is of relevance for the institution and/or early discontinuation of immunosuppressive drugs. ${ }^{25}$ In this questionnaire, over two-thirds of the participants reported starting prednisolone in case of acute minocycline-induced AIH without liver failure, whereas just under one-third would institute immunosuppressive therapy only in case of progression. In contrast to idiopathic AIH, most participants reported aiming for early withdrawal with or without histological evaluation of disease activity at three or six months. Only upon reoccurrence of symptoms and inflammatory activity most participants reported initiating further induction therapy followed by maintenance agents.

In conclusion, prednisolone remains the preferred first-line induction agent in JAILD, and MRC at presentation is performed by the large majority of respondents, indicating awareness of the recently defined nosological entity of ASC. Within the paediatric members of the IAIHG there is considerable experience with second-line therapeutic agents, which is largely concentrated in transplant centres.

\section{Acknowledgements}

Author contributions include: G.M.V.: original idea for the study; Y.S.d.B. and R.L.: study design, analysis of the data, drafting of the manuscript; D.V. and G.M.V.: study design and supervision. All authors approved the final version of the manuscript.

Full list of International Autoimmune Hepatitis Group contributors by response: Alexander Miethke, Bjorn 
Fischler, Carolina Jimenez-Rivera, Cigdem Arikan, Deidre Kelly, Denise Herzog, Dominique Debray, Efi Broide, Ekkehard Sturm, Etienne Sokal, Fernando Alvarez, Florence Lacaille, Florian Brinkert, Gilda Porta, Giuseppe Indolfi, Isabel Goncalves, Jia-Feng Wu, Jorg Jahnel, Jorge Amil Dias, Juan Cristobal Gana, Lorenzo D'Antiga, Loreto Hierro, Marek Woynarowski, Margarita Ramonet, Marianne Jørgensen, Miriam Cuarterolo, Mirta Ciocca, Nanda Kerkar, Nedim Hadzic, Oslem Durmaz, Peter Whitington, Pietro Vajro, Raffaele Iorio, Regino Gonzalez-Peralta, Richard Schreiber, Simon Ling, Susana Lopez, Thierry Lamireau, Tomoo Fujisawa, Tudor Pop, Ulrich Baumann, Uzma Shah, Valerie McLin.

\section{Declaration of conflicting interests \\ None declared.}

\section{Funding}

This study was supported by the United European Gastroenterology LINK award 2015.

\section{Ethics approval}

This study was conducted according to the Declaration of Helsinki.

\section{Informed consent}

This study does not contain any patient data. It is based on a questionnaire survey. We therefore did not seek ethical approval from an institutional review board or ethical committee nor asked for separate signed informed consent from the participants. The manuscript does not concern patientbased research, so it does not require ethical approval or informed consent.

\section{ORCID iD}

Ynto Sjoerd de Boer (D) http://orcid.org/0000-0002-4066-7593

\section{References}

1. Liberal R, Vergani D and Mieli-Vergani G. Paediatric autoimmune liver disease. Dig Dis 2015; 33(Suppl 2): 36-46.

2. Carbone M and Neuberger JM. Autoimmune liver disease, autoimmunity and liver transplantation. J Hepatol 2014; 60: $210-223$.

3. Krawitt EL. Autoimmune hepatitis. N Engl J Med 2006; 354: 54-66.

4. Gregorio GV, Portmann B, Reid F, et al. Autoimmune hepatitis in childhood: A 20-year experience. Hepatology 1997; 25: 541-547.

5. Mieli-Vergani G and Vergani D. Autoimmune hepatitis. Nat Rev Gastroenterol Hepatol 2011; 8: 320-329.

6. Vergani D, Alvarez F, Bianchi FB, et al. Liver autoimmune serology: A consensus statement from the committee for autoimmune serology of the International Autoimmune Hepatitis Group. J Hepatol 2004; 41: 677-683.

7. Chapman R, Fevery J, Kalloo A, et al. Diagnosis and management of primary sclerosing cholangitis. Hepatology 2010; 51: 660-678.
8. Gregorio GV, Portmann B, Karani J, et al. Autoimmune hepatitis/sclerosing cholangitis overlap syndrome in childhood: A 16-year prospective study. Hepatology 2001; 33: 544-553.

9. Liberal R, Grant CR, Ma Y, et al. CD39 mediated regulation of Th17-cell effector function is impaired in juvenile autoimmune liver disease. J Autoimmun 2016; 72: 102-112.

10. Mieli-Vergani G, Heller S, Jara P, et al. Autoimmune hepatitis. J Pediatr Gastroenterol Nutr 2009; 49: 158-164.

11. Alvarez F, Berg PA, Bianchi FB, et al. International Autoimmune Hepatitis Group Report: Review of criteria for diagnosis of autoimmune hepatitis. J Hepatol 1999; 31: 929-938.

12. Hennes EM, Zeniya M, Czaja AJ, et al. Simplified criteria for the diagnosis of autoimmune hepatitis. Hepatology 2008; 48: 169-176.

13. Manns MP, Czaja AJ, Gorham JD, et al. Diagnosis and management of autoimmune hepatitis. Hepatology 2010; 51: 2193-2213.

14. European Association for the Study of the Liver. EASL Clinical Practice Guidelines: Autoimmune hepatitis. J Hepatol 2015; 63: 971-1004.

15. Mieli-Vergani G, Vergani D, Baumann U, et al. Diagnosis and management of paediatric autoimmune liver disease: ESPGHAN Hepatology Committee Position Statement. $J$ Pediatr Gastroenterol Nutr 2018; 66: 345-360.

16. Manns MP, Woynarowski M, Kreisel W, et al. Budesonide induces remission more effectively than prednisone in a controlled trial of patients with autoimmune hepatitis. Gastroenterology 2010; 139: 1198-1206.

17. Woynarowski M, Nemeth A, Baruch Y, et al. Budesonide versus prednisone with azathioprine for the treatment of autoimmune hepatitis in children and adolescents. J Pediatr 2013; 163: 1347-1353.e1.

18. Liberal R, de Boer YS, Andrade RJ, et al. Expert clinical management of autoimmune hepatitis in the real world. Aliment Pharmacol Ther 2017; 45: 723-732.

19. Zizzo AN, Valentino PL, Shah PS, et al. Second-line agents in pediatric patients with autoimmune hepatitis: A systematic review and meta-analysis. $J$ Pediatr Gastroenterol Nutr 2017; 65: 6-15.

20. Ang GS, Simpson SA and Reddy AR. Mycophenolate mofetil embryopathy may be dose and timing dependent. Am J Med Genet A 2008; 146A: 1963-1966.

21. Perez-Aytes A, Marin-Reina P, Boso V, et al. Mycophenolate mofetil embryopathy: A newly recognized teratogenic syndrome. Eur J Med Genet 2017; 60: 16-21.

22. Garner SE, Eady A, Bennett C, et al. Minocycline for acne vulgaris: Efficacy and safety. Cochrane Database Syst Rev 2012; CD002086.

23. Lan J, Lahoti A and Lew DB. A severe case of minocycline-induced DRESS resulting in liver transplantation and autoimmune sequelae. Ann Allergy Asthma Immunol 2016; 116: 367-368.

24. Eichenfield AH. Minocycline and autoimmunity. Curr Opin Pediatr 1999; 11: 447-456.

25. de Boer YS, Kosinski AS, Urban TJ, et al. Features of autoimmune hepatitis in patients with drug-induced liver injury. Clin Gastroenterol Hepatol 2017; 15: 103-112.e2. 